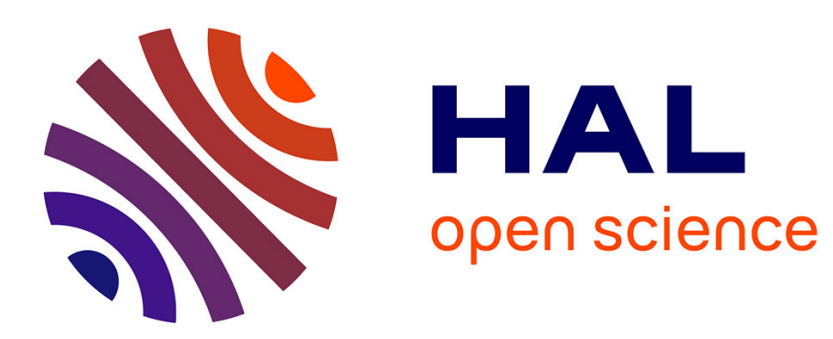

\title{
Flow and sound fields of low-Reynolds-number temporal jets at Mach numbers from 0.3 to 2
}

Christophe Bogey, Pierre Pineau

\section{To cite this version:}

Christophe Bogey, Pierre Pineau. Flow and sound fields of low-Reynolds-number temporal jets at Mach numbers from 0.3 to 2. 2018 AIAA/CEAS Aeroacoustics Conference, Jun 2018, Atlanta, United States. 10.2514/6.2018-3615 . hal-02352088

\section{HAL Id: hal-02352088 \\ https://hal.science/hal-02352088}

Submitted on 6 Nov 2019

HAL is a multi-disciplinary open access archive for the deposit and dissemination of scientific research documents, whether they are published or not. The documents may come from teaching and research institutions in France or abroad, or from public or private research centers.
L'archive ouverte pluridisciplinaire HAL, est destinée au dépôt et à la diffusion de documents scientifiques de niveau recherche, publiés ou non, émanant des établissements d'enseignement et de recherche français ou étrangers, des laboratoires publics ou privés. 


\title{
Flow and sound fields of low-Reynolds-number temporal jets at Mach numbers from 0.3 to 2
}

\author{
Christophe Bogey* and Pierre Pineau ${ }^{\dagger}$ \\ Laboratoire de Mécanique des Fluides et d'Acoustique \\ UMR CNRS 5509, Ecole Centrale de Lyon, Univ Lyon \\ 69134 Ecully, France
}

\begin{abstract}
Five temporally-developing isothermal round jets at a diameter-based Reynolds number $\operatorname{Re}_{D}$ of 3,125 and Mach numbers $M$ of $0.3,0.6,0.9,1.3$ and 2 have been computed using direct numerical simulation. The flow and near acoustic fields of the jets are described in detail, and cross-correlations between the two fields are presented in order to identify noise generation mechanisms, and especially that associated with the jet potential-core closing. That closing is found to occur later at a higher Mach number. Apart from that, the jet flow properties, including velocity spectra, are similar, and even nearly identical for $M \leq 1.3$. The jets appear to radiate acoustic waves mainly in the downstream direction, with amplitude increasing and wavelength decreasing with the Mach number. More quantitatively, the noise spectra nearly collapse when they are plotted as as a function of $k_{z} r_{0} \mathrm{M}^{-1}$, where $k_{z}$ is the axial wavenumber and $r_{0}$ is the initial jet radius, and scaled in amplitude using a $\mathrm{M}^{8}$ power law. The angle of sound emission is noted to decrease monotonically as time passes, except for the jet at $M=2$ for which it is close to the angle expected for Mach waves over a long time period. Finally, significant values of correlations are obtained between the pressure waves propagating downstream in the jet near field and the flow fluctuations on the jet axis near the time of potential-core closing for $M \geq 0.6$, with levels strengthening with the Mach number. The correlations calculated from the flow fluctuations at $r=r_{0}$ are much weaker. For the jet at $\mathrm{M}=2$, however, they suggest the generation of Mach waves in the mixing layers. These results are comparable to those reported for spatially-developing jets.
\end{abstract}

\section{Introduction}

For more than sixty years of research, noise generation in jet flows has been investigated in a large amount of theoretical, experimental and numerical studies. Sound sources have been described in several manners involving different concepts, such as acoustic quadrupoles, self noise and shear noise, instability waves, fine-scale turbulence and flow coherent structures, among others. Significant progress have thus been made, in particular for supersonic jets. ${ }^{1}$ Some questions, however, remains about the nature of the strong mixing noise component prevailing in the downstream direction, which is typically centered around a Strouhal number of $\mathrm{St}_{D}=f D / u_{j}=0.15$, where $f, D$ and $u_{j}$ are the frequency, and the jet diameter and velocity. ${ }^{2}$ This component has properties quite distinct from those of the omnidirectional, broadband mixing noise component dominant in the upstream and sideline directions. ${ }^{3}$ It seems to be generated by large-scale structures and/or instability waves over a wide range of Mach numbers, ${ }^{4}$ typically from $\mathrm{M}=u_{j} / c_{0}=0.5$ up to $\mathrm{M}=2$, where $c_{0}$ is the speed of sound in the ambient medium. On the basis on noise source localization ${ }^{5-7}$ and flow-noise cross-correlation ${ }^{8-10}$ results, it also appears to be produced at the end of the jet potential core where the shear layers merge, by a mechanism which is still not clearly understood but does not depend much on the Reynolds number. ${ }^{11}$

In order to get new insights on that mechanism, two temporally-developing subsonic round jets have been simulated in a recent study by the first author of this paper. ${ }^{12}$ The simulations of temporal flows

${ }^{*}$ CNRS Research Scientist, AIAA Senior Member \& Associate Fellow, christophe.bogey@ec-lyon.fr

${ }^{\dagger} \mathrm{PhD}$ student, pierre.pineau@doctorant.ec-lyon.fr 
are relatively rare, but quite a few have been performed in the past to explore acoustic sources in mixing layers. ${ }^{13-16}$ In our previous study, two temporal jets at a Mach number of 0.9 and diameter-based Reynolds numbers of 3,125 and 12,500 have been computed. The jet at a Reynolds number of 12,500 develops more rapidly, exhibits more fine turbulent scales, and generates more high-frequency acoustic waves than the jet at a Reynolds number of 3,125, as expected. In both cases, however, the flow fluctuations on the jet axis are strongly intermittent at the time of potential-core closing, and low-frequency acoustic waves are subsequently radiated in the downstream direction. Furthermore, the centerline flow fluctuations and these waves are found to be strongly correlated, as observed for spatially-developing jets. This led us to calculate conditional averages of the jet near fields using a sampling synchronization with the minimum values of centerline axial velocity at the time of potential-core closing. The presence of a velocity deficit and a vorticity excess on the jet axis at that time, and the consecutive emission of sound waves are clearly visible in the resulting flow and pressure fields.

Given these results, a logical next step is to deal with temporally-developing jets at different Mach numbers to examine the influence of the velocity on the jet development and noise generation. The variations of jet acoustic characteristics with the Mach number have been investigated in a number of studies for spatially-developing jets in order to obtain information on noise components and their associated sources. The theoretical work of Lighthill ${ }^{17}$ and Ffowcs Williams ${ }^{18}$ established, for instance, that the overall sound pressure level should increase with the eighth power of velocity for subsonic jets, but with the third power of velocity for supersonic jets. Overall, these power laws apply to jet noise measurements, although the power-law exponent appears to depend on the radiation angle. ${ }^{2}$ In particular, there is a rapid increase of the exponent as the angle decreases, ${ }^{3}$ suggesting the presence of a specific noise component in the jet flow direction. As for the sound spectra, they are found to scale as the Strouhal number $\mathrm{St}_{D}$, except maybe ${ }^{19}$ for very low angles where scalings as the Helmholtz number $\mathrm{Hm}=f D / c_{0}$ with $^{20}$ or without ${ }^{21}$ a correction by a Doppler factor have been reported. Finally, it can be noted that for jets at $\mathrm{M} \simeq 2$, the levels of crosscorrelations between sound pressure fluctuations in the downstream direction and flow fluctuations around the end of the potential core are high, but that they sharply decrease at lower Mach numbers, and become even negligible for low-subsonic jets. ${ }^{10}$

In the present paper, the flow and acoustic fields obtained for five temporally-developing isothermal round jets by solving the unsteady compressible Navier-Stokes equations using high-order finite-difference schemes are presented. The jets are at a diameter-based Reynolds number of 3,125 and at Mach numbers of $0.3,0.6,0.9,1.3$ and 2 . The jet at Mach 0.9 is one of the temporal jets mentioned above, ${ }^{22}$ and the jet at Mach 2 was considered in a recent $\operatorname{study}^{23}$ to explore the generation of shocked waves in the vicinity of supersonic jets. Here, the objective is to carefully describe the flow and sound fields of the jets in order to determine whether the observations made for the jet at Mach 0.9 are valid for the others. In particular, we will discuss whether the five jets all produce low-frequency sound waves in the downstream direction around the time of potential-core closing, which is not obvious for the low subsonic and the high supersonic jets at $\mathrm{M}=0.3$ and 2 . As in previous work, cross-correlations will be computed between flow quantities and sound pressure outside the jet in order to track causal links between sources and observer. Special attention will be paid to the flow fluctuations on the jet centerline, where intermittency is expected to be strong. Naturally, the scalings and variations of the results with the Mach number will be compared to those available in the literature for spatially-developing jets.

The paper is organized as follows. The main characteristics of the jets and of the simulations, including initial conditions, numerical methods, grid and computational parameters, are documented in section II. The simulation results, namely vorticity and pressure snapshots, the main properties of the jet velocity and near pressure fields, and flow-noise cross-correlations are shown in section III. Finally, concluding remarks are given in section IV.

\section{Parameters}

\section{A. Jet definition}

The five jets in this work are round and isothermal, and have a Reynolds number of $\operatorname{Re}_{D}=u_{j} D / \nu=3,125$ and Mach numbers of $\mathrm{M}=0.3,0.6,0.9,1.3$ and 2 , where $u_{j}$ and $D=2 r_{0}$ are the jet initial centerline velocity and diameter, and $\nu$ is the kinematic molecular viscosity. The ambient temperature and pressure are equal to $T_{0}=293 \mathrm{~K}$ and $p_{0}=10^{5} \mathrm{~Pa}$. At initial time $t=0$, the hyperbolic-tangent profile of axial velocity presented in figure 1 (a) is imposed. Following the variations of $\delta_{\theta} / r_{0}$ with the Reynolds number 
observed in experiments for initially laminar jets, ${ }^{24}$ the momentum thickness of the mixing layer is fixed at $\delta_{\theta}=0.0358 r_{0}$. This leads to the initial momentum Reynolds number of $\operatorname{Re}_{\theta}=u_{j} \delta_{\theta} / \nu=56$. Radial and azimuthal velocities are set to zero, pressure is equal at $p_{0}$, and density is determined by a Crocco-Busemann relation.

(a)

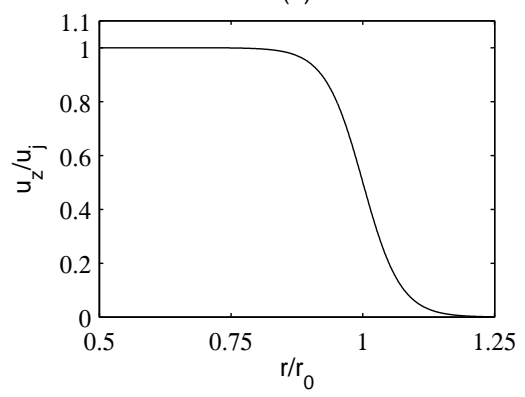

(b)

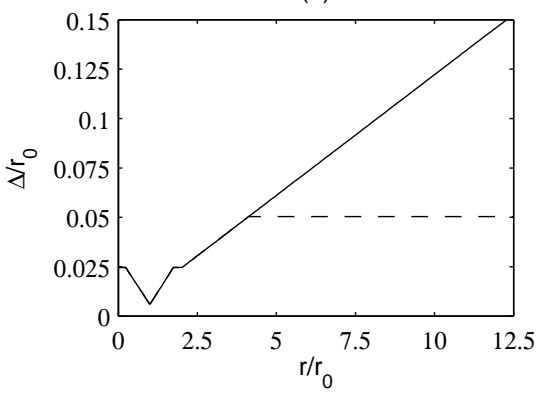

Figure 1. Radial profiles (a) of axial velocity $u_{z} / u_{j}$ at $t=0$ and (b) of radial spacing $\Delta r / r_{0}$ for $M=0.3,0.6,0.9$ and 1.3 and $---M=2$.

At $t=0$, velocity disturbances of low amplitude are added in the mixing layers in order to seed the laminar-turbulent transition. For this, divergence-free Gaussian ring vortices of radius $r_{0}$ are introduced. ${ }^{25}$ These vortices have a half-width of $2 \delta_{\theta}$, and are regularly distributed in the axial direction every $\Delta z=$ $0.025 r_{0}$, where $\Delta z$ is the axial mesh spacing. At each position, the vortex has a maximum velocity randomly fixed between 0 and $0.01 u_{j}$, and is weighted in the azimuthal direction by the function $\cos \left(n_{\theta} \theta+\varphi\right)$ where $n_{\theta}$ and $\varphi$ are randomly chosen between 0 and 32 and between 0 and $2 \pi$, respectively. The same forcing strength is chosen for the jets at $\mathrm{M}=0.3,0.6,0.9$ and 1.3 to arbitrarily reach a peak turbulence intensity close to $1 \%$ at $t=0$. The use of such an initial condition for the jet at $\mathrm{M}=2$ was found to lead to a laminar-turbulent transition process much longer than $100 r_{0} / u_{j}$, which would be very costly. The forcing strength is therefore higher for the fifth jet, resulting to an initial turbulence intensity of $4 \%$ in that case. Finally, note that several runs are performed for each jet using different random seeds in order to improve the statistical convergence of the results.

\section{B. Numerical methods}

The numerical framework is identical to that used in recent simulations of round spatial ${ }^{26-29}$ and temporal $^{12,23}$ jets. The simulations are carried out using an in-house solver of the three-dimensional filtered compressible Navier-Stokes equations in cylindrical coordinates $(r, \theta, z)$ based on low-dissipation and lowdispersion, high-order explicit schemes. The axis singularity is taken into account by the method of Mohseni \& Colonius. ${ }^{30}$ In order to alleviate the time-step restriction near the cylindrical origin, the derivatives in the azimuthal direction around the axis are calculated at coarser resolutions than permitted by the grid. ${ }^{31}$ For the points closest to the jet axis, they are evaluated using 16 points, yielding an effective resolution of $2 \pi / 16$. Fourth-order eleven-point centered finite differences are used for spatial discretization, and a secondorder six-stage Runge-Kutta algorithm is implemented for time integration. ${ }^{32}$ A twelfth-order eleven-point centered filter is applied explicitly to the flow variables every time step in order to remove grid-to-grid oscillations while leaving larger scales mostly unaffected. Non-centered finite differences and filters are also used near the grid boundaries. ${ }^{33,34}$ The radiation conditions of Tam \& Dong ${ }^{35,36}$ are applied at the sideline boundaries to avoid significant acoustic reflections. Since a temporally-developing flow is considered, periodic boundary conditions are imposed in the axial direction.

\section{Simulation parameters}

The main grid and simulation parameters are provided in table 1 , and the mesh spacings are presented in figure 1(b). The grids used contain 980 million points, with $n_{r}=382, n_{\theta}=512$ and $n_{z}=4800$ for the jet at $\mathrm{M}=0.9, n_{r}=382, n_{\theta}=256$ and $n_{z}=4800$ for $\mathrm{M}=0.3,0.6$ and 1.3 , and $n_{r}=382, n_{\theta}=256$ and $n_{z}=9600$ for $\mathrm{M}=2$. They extend up to $L_{z}=120 r_{0}$ and out to $L_{r}=30 r_{0}$ in the first case, $L_{z}=240 r_{0}$ and $L_{r}=30 r_{0}$ in the second one, and $L_{z}=240 r_{0}$ and $L_{r}=13 r_{0}$ in the third one. The mesh spacing in the axial direction is uniform and equal to $\Delta z=0.025 r_{0}$, whereas the mesh spacing in the radial direction 
varies. The latter is minimum and equal to $\Delta r=0.006 r_{0}$ at $r=r_{0}$. It is maximum and equal to $\Delta r=0.2 r_{0}$ for $r \geq 16 r_{0}$ for the jets at $\mathrm{M} \leq 1.3$ and to $\Delta r=0.05 r_{0}$ for $r \geq 4 r_{0}$ for the jet at $\mathrm{M}=2$, yielding normalized wavenumbers of $k r_{0}=7.8$ and 31 , respectively, for a wave discretized by four points per wavelength. The mesh is finer for the Mach 0.2 jet in order to allow for the propagation of shocked waves in the jet near field in that case. ${ }^{23}$ The use of $n_{\theta}=256$ and 512 points in the azimuthal direction leads to $r \Delta \theta=0.024 r_{0}$ and $0.012 r_{0}$ at $r=r_{0}$. Remark that the simulations have been checked to be fully-resolved DNS from the calculation of the turbulent kinetic energy budgets.

The computations are performed using an OpenMP-based in-house solver on 32-core nodes of Intel E54650 processors with a clock speed of $2.7 \mathrm{GHz}$ and 16-core nodes of Intel E5-2670 processors at 2.6 GHz. The total number of iterations is equal to 18,000 for $\mathrm{M}=0.3$ and $0.6,23,000$ for $\mathrm{M}=0.9,23,000$ for $\mathrm{M}=1.3$, and 21,000 for $\mathrm{M}=2$, leading to final times of $t_{\max } u_{j} / r_{0}$ of $50,60,75,75$ and 96 , respectively. The time step $\Delta t$ is chosen so that $\Delta t=\mathrm{CFL} \times \Delta r\left(r=r_{0}\right) / c_{0}$, where $\mathrm{CFL}=1.5$ for $\mathrm{M}=0.3,0.9$ for $\mathrm{M}=0.6$, 0.6 for $\mathrm{M}=0.9,0.55$ for $\mathrm{M}=1.3$ and 0.37 for $\mathrm{M}=2$, in order to ensure the stability of the explicit time integration. For the present grids of approximately one billion points, 200 GB of memory are required, and about 1,000 CPU hours are consumed for 1,000 iterations. Density, the three velocity components, pressure and vorticity norm are recorded on the jet axis at $r=0$ and on the cylindrical surfaces at $r=r_{0}, 4 r_{0}$ and $20 r_{0}$, at a sampling frequency allowing spectra to be computed up to $\mathrm{St}_{D}=10$, and on the four azimuthal planes at $\theta=0, \pi / 2, \pi$ and $3 \pi / 2$, at half the frequency mentioned above. For each jet, several runs, namely ten runs for $\mathrm{M}=0.9$, five runs for $\mathrm{M}=0.3,0.6$ and 1.3, and four runs for $\mathrm{M}=2$, are executed using different random seeds. The statistical results obtained in each run are averaged over the periodic directions $z$ and $\theta$, and are then ensemble averaged, providing mean values denoted by $\langle.>$.

Table 1. Jet Mach number M, number of runs $n_{\text {runs }}$, extents of the computational domain in the axial and radial directions $L_{z}$ and $L_{r}$, total simulation time $t_{\max }$, and time of potential-core closing $t_{c}$.

\begin{tabular}{cccccc}
\hline $\mathrm{M}$ & $n_{\text {runs }}$ & $L_{z} / r_{0}$ & $L_{r} / r_{0}$ & $t_{\max } r_{0} / u_{j}$ & $t_{c} r_{0} / u_{j}$ \\
\hline 0.3 & 5 & 240 & 30 & 50 & 16.9 \\
0.6 & 5 & 240 & 30 & 60 & 18.5 \\
0.9 & 10 & 120 & 30 & 75 & 21.6 \\
1.3 & 5 & 240 & 30 & 75 & 30.1 \\
2 & 4 & 240 & 13 & 96 & 48.9 \\
\hline
\end{tabular}

\section{Results}

\section{A. Vorticity and pressure snapshots}

Snapshots of the vorticity norm and pressure fluctuations obtained in the $(z, r)$ plane at $t u_{j} / r_{0}=25,30,40$ and 50 for the jets at $\mathrm{M}=0.3,0.6,0.9$ and 1.3 , respectively, and at $t u_{j} / r_{0}=75$ for the jet at $\mathrm{M}=2$, are represented in figures 2 and 3 . At these times, the jets are developed and exhibit in the whole radial section vortical structures, whose strength, normalized by the initial jet velocity, seems to decrease with the Mach number. At earlier times, in all cases, following the growth of instability waves in the hyperbolic-tangent velocity profile, ${ }^{37}$ vortices have rolled up and interacted with each other in the initially laminar shear layers, as shown in previous papers ${ }^{12,23}$ for the jets at $\mathrm{M}=0.9$ and 2 . Then, the mixing layers have merged on the jet axis, resulting in the disappearance of the potential core. This occurs more slowly as the Mach number increases, as will be quantified later.

In the pressure fields, alternatively positive and negative fluctuations are visible in the immediate vicinity of the jets, especially at low Mach numbers. They correspond to the aerodynamic pressure disturbances induced by the large turbulent structures of the flow. ${ }^{38,39}$ Farther from the jet axis, acoustic waves appear to propagate in the downstream direction, as observed at shallow angles for spatially-developing subsonic jets. $^{25,40}$ In all cases, they seem to be mostly symmetric with respect to the jet centerline, and to have a large spatial extent along the wave front direction. Their levels strongly increase with the Mach number, ranging approximately from about $5 \mathrm{~Pa}$ at $\mathrm{M}=0.3$ up to about $3000 \mathrm{~Pa}$ at $\mathrm{M}=2$. In addition, their associated wavelengths decreases with the jet velocity. For instance, they are typically equal to $20 r_{0}, 15 r_{0}$ and $10 r_{0}$ at $\mathrm{M}=0.6,0.9$ and 1.3 , respectively. Finally, the shape of these waves is clearly circular for the 

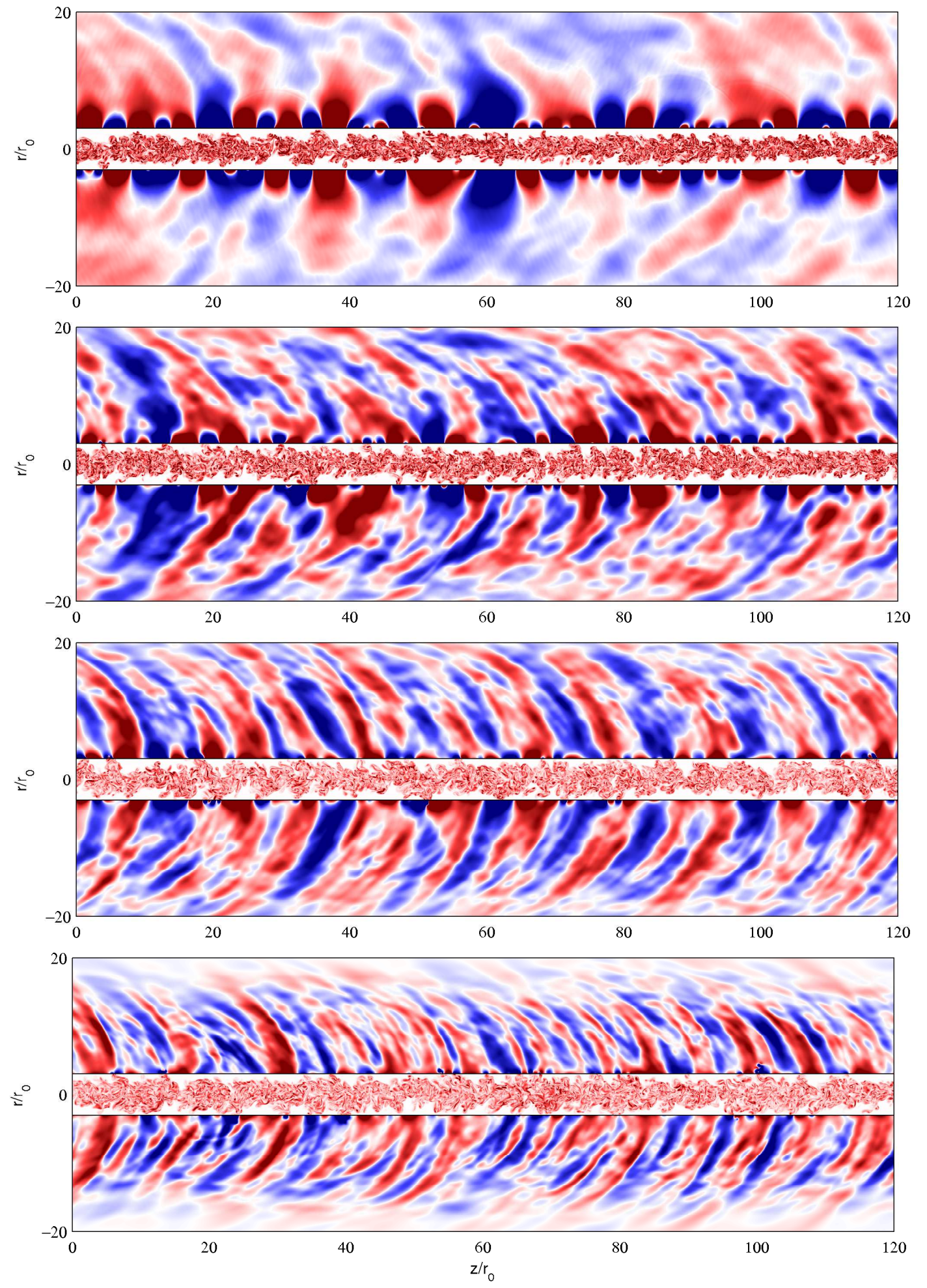

Figure 2. Representation of vorticity norm for $r \leq 3 r_{0}$ and of pressure fluctuations otherwise, obtained for $M=0.3,0.6,0.9$ and 1.3 , from top to bottom, at $t u_{j} / r_{0}=25,30,40$ and 50 , respectively. The color scales range up to the level of $5 u_{j} / r_{0}$ for vorticity, and from -5 to $5 \mathrm{~Pa},-40$ to $40 \mathrm{~Pa},-200$ to $200 \mathrm{~Pa}$, and -800 to $800 \mathrm{~Pa}$ for pressure, from blue to red. 


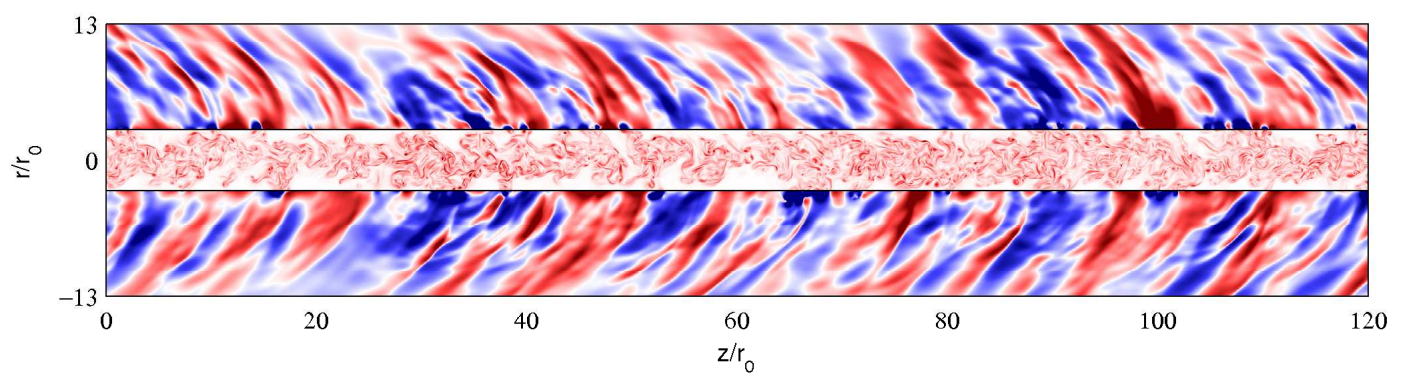

Figure 3. Vorticity and pressure fluctuations obtained for $M=2$ at $t u_{j} / r_{0}=75$. The color scales range up to the level of $4 u_{j} / r_{0}$ for vorticity, and from -3000 up to $3000 \mathrm{~Pa}$ for pressure, from blue to red.

four jets at $\mathrm{M} \leq 1.3$, but rather planar for the jet at $\mathrm{M}=2$, suggesting the generation of Mach waves in that highly-supersonic case.

\section{B. Properties of the velocity fields}

The time variations of the shear-layer momentum thickness and of the mean centerline axial velocity are presented as a function of $t u_{j} / r_{0}$ in figures $4(\mathrm{a}-\mathrm{b})$. The shear layer spreads more slowly and the jet develops at a later time at a higher Mach number. As a result, the mean centerline velocity reaches a value of $0.95 u_{j}$ at $t_{c} u_{j} / r_{0}=16.9$ for $\mathrm{M}=0.3,18.5$ for $\mathrm{M}=0.6,21.6$ for $\mathrm{M}=0.9,30.1$ for $\mathrm{M}=1.3$ and 48.9 for $\mathrm{M}=2$, as reported in table 1 . This is expected given the reduction of the growth rate of instability waves in initially laminar mixing layers with increasing Mach number. ${ }^{41}$ In order to examine the possible differences in jet development after the potential-core closing, the mean centerline axial velocities are replotted as a function of $\left(t-t_{c}\right) u_{j} / r_{0}$ in figure 4(c). The two curves obtained for $\mathrm{M}=0.3$ and 0.6 are superimposed. The other curves deviate from them, almost imperceptibly for $\mathrm{M}=0.9$, appreciably for $\mathrm{M}=1.3$ and strongly for $\mathrm{M}=2$. The velocity decay is therefore weaker as the jet velocity increases, especially for Mach numbers well above 1 , which can be attributed to compressibility effects. ${ }^{42}$

(a)

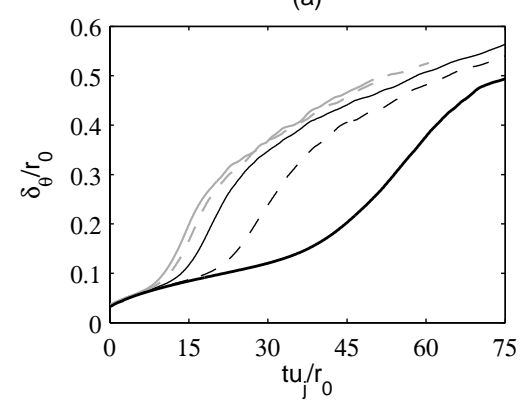

(b)

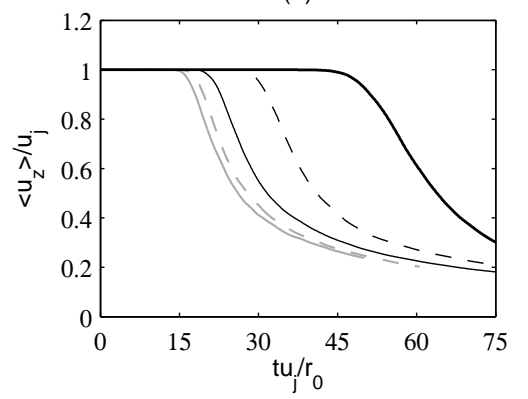

(c)

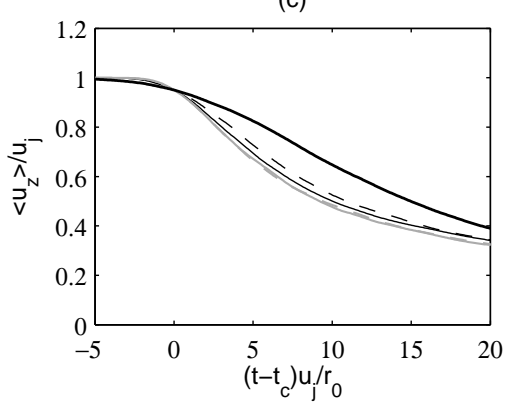

Figure 4. Time variations (a) of shear-layer momentum thickness $\delta_{\theta} / r_{0}$ and (b) of mean axial velocity $<u_{z}>/ u_{j}$ at $r=0$ as a function of $t u_{j} / r_{0}$, and (c) of $\left\langle u_{z}\right\rangle / u_{j}$ at $r=0$ as a function of $\left(t-t_{c}\right) u_{j} / r_{0}:-M=0$, $\mathrm{M}=\mathbf{0 . 6},-\mathrm{M}=0.9,--\mathrm{M}=1.3,-\mathrm{M}=\mathbf{2}$.

The time variations of the axial turbulence intensities at $r=0$ and $r=r_{0}$ are shown as a function of $\left(t-t_{c}\right) u_{j} / r_{0}$ in figures $5(\mathrm{a}-\mathrm{b})$. As for the mean centerline density in figure 4(c), the results are similar for $\mathrm{M} \leq 1.3$, but differ for $\mathrm{M}=2$. For $\mathrm{M} \leq 1.3$, the turbulence intensities reach peaks of about $17 \%$ at $t \simeq t_{c}+4 r_{0} / u_{j}$ on the jet axis, and of about $20 \%$ near the time of potential-core closing in the mixing layers. For $\mathrm{M}=2$, the peak values are obtained later, at $t=t_{c}+8.1 r_{0} / u_{j}$ and at $t \simeq t_{c}+2.8 r_{0} / u_{j}$, and they are equal to $19.4 \%$ and $19.5 \%$, respectively. Moreover, after the peaks, the decrease of the turbulent intensities is less pronounced for the jet at $\mathrm{M}=2$ than for the others, in agreement with the slower jet flow development in the former case that in the latter.

The spectra of axial velocity fluctuations, normalized by the jet velocity, obtained at $r=0$ at $t=$ $t_{c}+5 r_{0} / u_{j}$ and at $r=r_{0}$ at $t=t_{c}$ are represented in figures $6(\mathrm{a}-\mathrm{b})$ as a function of axial wavenumber $k_{z} r_{0}$. In both cases, the spectra for $0.3 \leq \mathrm{M} \leq 1.3$ are close to one another, suggesting similar spatial arrangements of the flow structures over this wide range of Mach numbers. In particular, the spectra at $r=0$ peak 
(a)

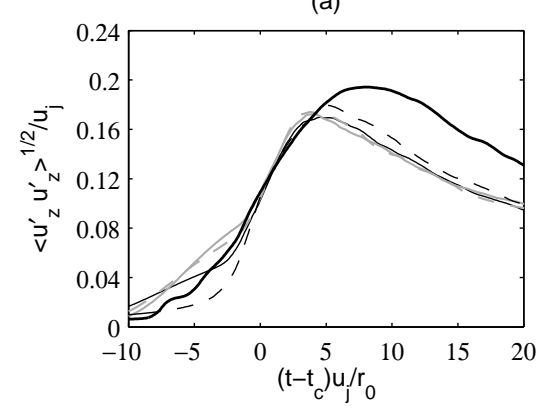

(b)

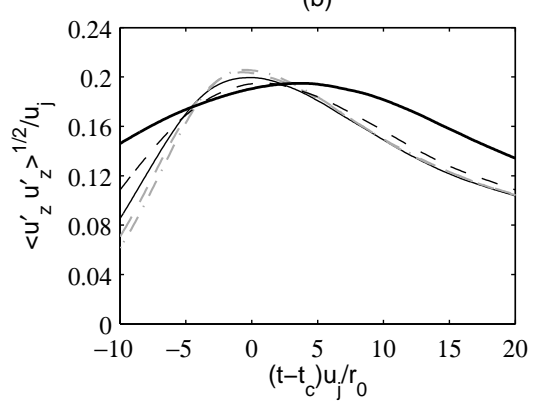

Figure 5. Time variations of the rms values of axial velocity fluctuations $u_{z}^{\prime}$ at (a) $r=0$ and (b) $r=r_{0}$ as a function of $\left(t-t_{c}\right) u_{j} / r_{0}:-M=0.3,---M=0.6,-M=0.9,--M$ $\mathbf{M}=\mathbf{2}$.

approximately at $k_{z} r_{0}=0.4$, yielding a wavelength $\lambda_{z} \simeq 15 r_{0}$ in the axial direction on the jet centerline. As the Mach number increases, however, the magnitude of the flow components tends to increase at lower wavenumbers, especially for $k_{z} r_{0} \leq 0.2$ at $r=0$, and to decrease at higher wavenumber. This trend is much more marked for the jet at $\mathrm{M}=2$. Therefore, at a higher velocity, the jet flow exhibits stronger large-scale structures and weaker fine scales. This provides an explanation for the apparent reduction of the vorticity levels with the Mach number in the snapshots of figures 2 and 3.

(a)

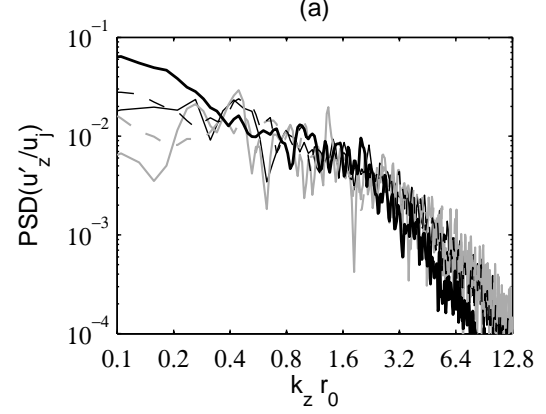

(b)

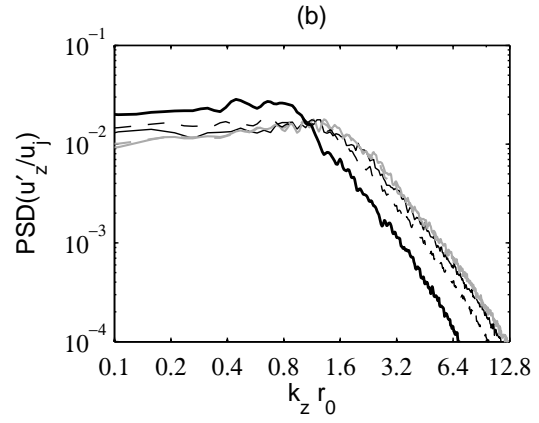

Figure 6. Representation of the power spectral densities (PSD) of axial velocity fluctuations $u_{z}^{\prime} / u_{j}$ obtained at (a) $r=0$ at $t=t_{c}+5 r_{0} / u_{j}$ and (b) $r=r_{0}$ at $t=t_{c}$ as a function of axial wavenumber $k_{z} r_{0}$ : $\mathrm{M}=0.3,--\mathrm{M}=0.6, \stackrel{-}{-} \mathrm{M}=0.9,---\mathrm{M}=1.3,-\mathrm{M}=2$.

\section{Properties of the pressure fields}

The time variations of the rms values of pressure fluctuations at $r=10 r_{0}$ from the jet axis are presented in figures $7(\mathrm{a}-\mathrm{b})$, normalized by $\mathrm{M}^{4}$. Strong peaks emerge after the time of potential-core closing. They are reached, for example, at $t=21.5 r_{0} / u_{j}=t_{c}+4.6 r_{0} / u_{j}$ for $\mathrm{M}=0.3$ and at $t=81.5=t_{c}+32.6 r_{0} / u_{j}$ for $\mathrm{M}=2$ in figure $7(\mathrm{a})$. More precisely, they are all obtained at $t \simeq t_{c}+15 r_{0} / c_{0}$ in figure $7(\mathrm{~b})$, where the results are plotted as a function of $\left(t-t_{c}\right) c_{0} / r_{0}$ by assuming a wave propagation at the ambient speed of sound. The peak values are very similar for the Mach numbers of $\mathrm{M}=0.6,0.9$ and 1.3 , indicating that the sound levels increase roughly with the eighth power of velocity as expected for subsonic jets. ${ }^{17}$ However, the peak for the jet at $M=2$ is significantly below the other ones, implying that, if any, the power law exponent is lower for the noise emitted by the high-supersonic jet.

The pressure spectra calculated at $r=10 r_{0}$ at $t=t_{c}+15 r_{0} / c_{0}$, i.e. close to the time of peak intensity, are provided in figures $8(\mathrm{a}-\mathrm{b})$. The increase in level and frequency of the acoustic waves with the jet Mach number is clearly visible in figure $8(\mathrm{a})$ where they are represented as a function of axial wavenumber $k_{z} r_{0}$. The dominant components shift, for instance, from $k_{z} r_{0} \simeq 0.12$ for $\mathrm{M}=0.3$ up to $k_{z} r_{0} \simeq 1$ for $\mathrm{M}=2$. On the contrary, the spectra strikingly resemble each other in figure 8(b) where they are plotted as a function of $k_{z} r_{0} \mathrm{M}^{-1}$ and normalized in amplitude using a $\mathrm{M}^{8}$ power law. They even collapse well for the jets at Mach numbers $\mathrm{M} \leq 1.3$, over the whole range of wavenumbers considered. The wavenumber scaling of the present spectra is thus similar to the $\mathrm{St}_{D}=f D / u_{j}$ frequency scaling obtained for spatially-developing jets. For the 
(a)

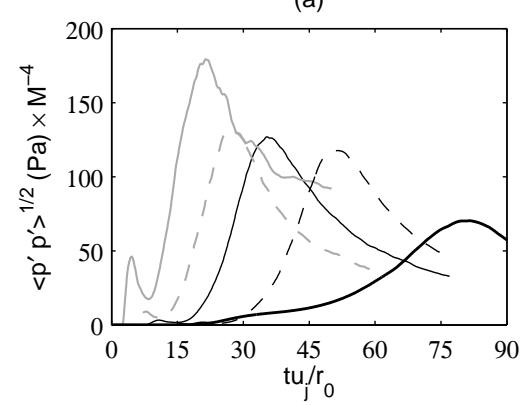

(b)

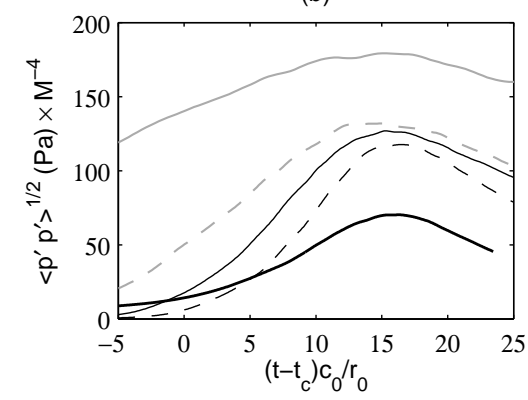

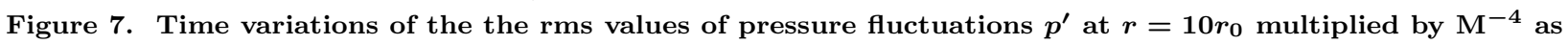
a function of (a) $t u_{j} / r_{0}$ and (b) $\left(t-t_{c}\right) c_{0} / r_{0}:-\mathrm{M}=0.3,---\mathrm{M}=0.6,-$

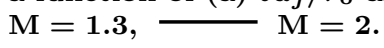

jet at $\mathrm{M}=2$, however, the spectrum shows differences, and is more strongly dominated by low-wavenumber components compared to the other spectra. These results are consistent with the Mach number dependence observed for the velocity spectra of figure 6 .
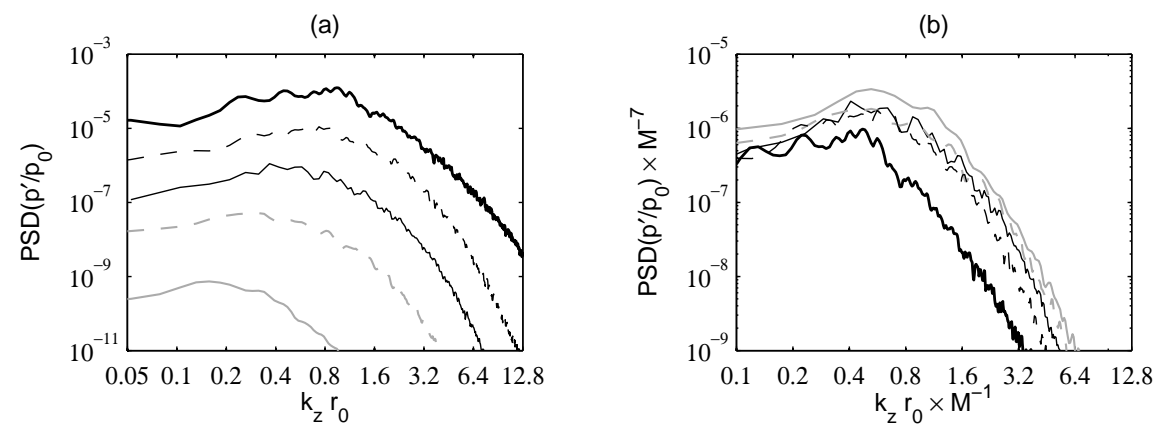

Figure 8. Representation of the power spectral densities (PSD) of pressure fluctuations $p^{\prime} / p_{0}$ obtained at $r=10 r_{0}$ at $t=t_{c}+15 r_{0} / c_{0}$; (a) PSD as a function of $k_{z} r_{0}$ and (b) PSD $\times \mathrm{M}^{-7}$ as a function of $k_{z} r_{0} \times \mathrm{M}^{-1}$. $\mathrm{M}=0.3,--\mathrm{M}=0.6,-\mathrm{M}=0.9,---\mathrm{M}=1.3,-2$.

In order to further characterize the jet acoustic field, the variations of the main radiation angle, estimated from pressure cross-correlations, and of the skewness factor of the pressure fluctuations at $r=10 r_{0}$ are displayed in figures $9(\mathrm{a}-\mathrm{b})$. In figure $9(\mathrm{a})$, the radiation angle decreases monotonically as time passes, except for the jet at $\mathrm{M}=2$ for which it remains near the angle $\phi=\cos ^{-1}\left(c_{0} / u_{c}\right)$ expected for Mach waves between $t \simeq t_{c}+10 r_{0} / c_{0}$ and $t \simeq t_{c}+16 r_{0} / c_{0}$. In the same way, in figure $9(\mathrm{~b})$, the skewness factor of pressure fluctuations at $r=10 r_{0}$ is close to zero for all jets except for the high-supersonic jet. In the latter case, the skewness factor is higher than 0.3 over a long time period due to the presence of shocked waves in the jet near field. ${ }^{23}$

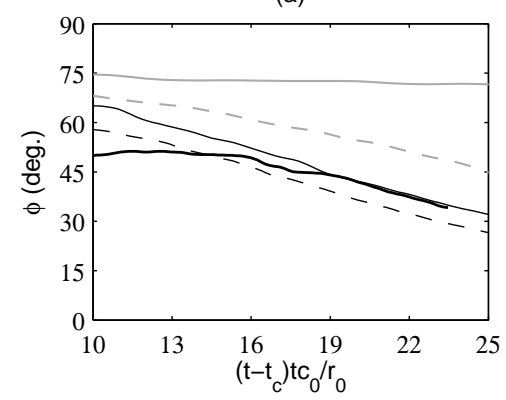

(b)

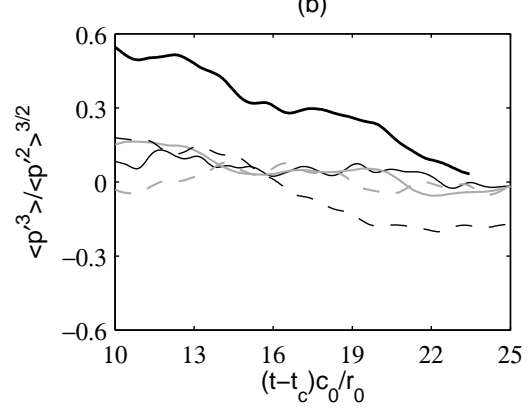

Figure 9. Time variations (a) of the radiation angle $\phi$, and (b) of the skewness factor of pressure fluctuations at $r=10 r_{0}:-\mathrm{M}=0.3,---\mathrm{M}=0.6,-\mathrm{M}=0.9,---\mathrm{M}=\mathbf{1 . 3},-$ 


\section{Flow-noise cross-correlations}

In order to identify links between the flow and sound fields, cross-correlations are computed between flow quantities in the jet and pressure outside, as was done in several experimental and numerical investigations for spatially-developing ${ }^{8-11,43-47}$ and temporally-developing ${ }^{12,23}$ jets. In the present work, correlations between pressure fluctuations $p^{\prime}$ at position $\left(r=r_{a c}, z\right)$ and time $t=t_{a c}$, and axial velocity fluctuations $u_{z}^{\prime}$ and vorticity fluctuations $|\omega|^{\prime}$ at $\left(r=r_{s}, z+\delta z\right)$ and time $t_{a c}-\delta t$ are calculated. For velocity $u_{z}^{\prime}$, for example, they are given by

$$
\mathcal{C}_{u_{z}^{\prime} p^{\prime}}(\delta z, \delta t)=\frac{\left\langle p^{\prime}\left(r_{a c}, \theta, z, t_{a c}\right) \times u_{z}^{\prime}\left(r_{s}, \theta, z+\delta z, t_{a c}-\delta t\right)\right\rangle}{\left\langle p^{\prime 2}\left(r_{a c}, \theta, z, t_{a c}\right)\right\rangle^{1 / 2} \times\left\langle u_{z}^{\prime 2}\left(r_{s}, \theta, z+\delta z, t_{a c}-\delta t\right)\right\rangle^{1 / 2}}
$$

with $r_{a c}=10 r_{0}, t_{a c}=t_{c}+22 r_{0} / c_{0}$, and $r_{s}=0$ and $r_{s}=r_{0}$ to consider correlations with flow fluctuations on the jet centerline and in the mixing layers.

The correlations maps determined from the centerline velocity and vorticity fluctuations are represented, respectively, in figures 10 and 11 for the five jets. The dashed line represents the time of potential-core closing, and the solid line indicates a propagation at the ambient speed of sound between the centerline and the near-field points. The results obtained for the four jets at $0.6 \leq \mathrm{M} \leq 2$ are very similar, suggesting the presence of common noise generation mechanisms over that range of Mach numbers. More precisely, the peak correlation values are found close to the intersection of the dashed and solid lines, for negative separation distances $\delta z$. This supports that the dominant acoustic waves are emitted in the downstream direction around the time of potential core-closing. The correlations are negative for $u_{z}^{\prime}$ and positive for $|\omega|^{\prime}$, which is likely due, as discussed in a previous paper, ${ }^{12}$ due to the occurrence of velocity deficit and vorticity excess on the jet axis at $t \simeq t_{c}$. The correlation levels, which do not exceed 0.15 at $\mathrm{M}=0.6$ and are greater than 0.25 for $\mathrm{M} \geq 1.3$, increase with the jet velocity, as observed for spatially-developing jets. ${ }^{10,11}$ Moreover, strong correlations are also encountered long after $t=t_{c}$ for the velocity fluctuations, but before $t=t_{c}$ for the vorticity fluctuations, except for the jet at $\mathrm{M}=0.6$ in that latter case. This persistence of significant correlations over a long period of time is related to the convection of the turbulent structures by the jet flow. ${ }^{12}$

For the jet at $\mathrm{M}=0.3$, spots of notable correlation are visible in figures 10 (a) and $11(\mathrm{a})$, but they do not appear to be consistent with a propagation at the speed of sound between a source and an observer point. The reason for that is currently unclear. One possibility is that the pressure fluctuations at $r=10 r_{0}$ from which the correlations are computed contain components of aerodynamic nature. ${ }^{38,39}$ Another possibility, which does not exclude the previous one, is that the sound source revealed in the four jets at $\mathrm{M} \geq 0.6$ cannot be detected in a jet at $\mathrm{M}=0.3$ because of its inefficiency at low Mach numbers. ${ }^{3}$

The correlations maps obtained from the axial velocity and vorticity fluctuations at $r=r_{0}$ for the three jets at $\mathrm{M} \geq 0.9$ are presented in figures 12 and 13 . The results for the jets at $\mathrm{M}=0.3$ and 0.6 are not shown because of negligible correlation levels in these two cases. As for the correlations evaluated from centerline flow disturbances in figures 10 and 11, the correlations in figures 12 and 13 also strengthen with the jet velocity. However, the peak correlation values are much lower than previously, and do not specifically emerge at the time of potential-core closing. Finally, significant correlations, aligned with the solid line indicating an acoustic propagation between the flow and near-field points, are seen in figure 12(c) for the jet at $\mathrm{M}=2$ for $t \leq t_{c}$. They can be attributed to the generation of Mach waves in the mixing layers of that highly-supersonic jet.

\section{Conclusion}

In this paper, the flow and the acoustic fields computed for temporally-developing isothermal round jets at a Reynolds number of 3,125 and Mach numbers of $0.3,0.6,0.9,1.3$ and 2 using direct numerical simulation are presented. Flow-noise cross-correlations are also shown to highlight connections between the two fields. Overall, despite the later closing of the jet potential core at a higher Mach number, the flow and sound fields of the jets exhibit very similar properties, especially for $M \leq 1.3$. In particular, the near-field pressure spectra are very close when they are plotted as a function of the axial wavenumber divided by the jet Mach number and normalized using the eighth power of velocity, as expected for spatially-developing subsonic jets. The jets at $\mathrm{M} \geq 0.6$ all radiate strong acoustic waves in the downstream direction around the time of potential-core closing. This is confirmed by the flow-noise cross-correlations calculated from centerline 
(a)

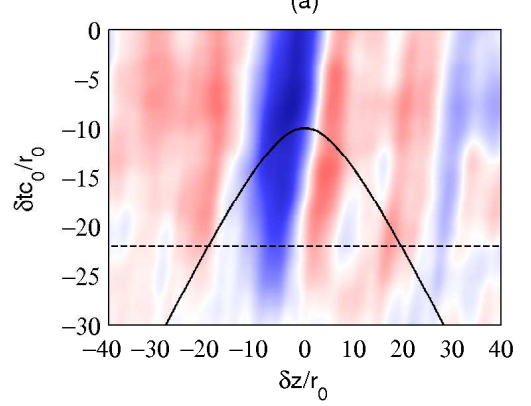

(b)

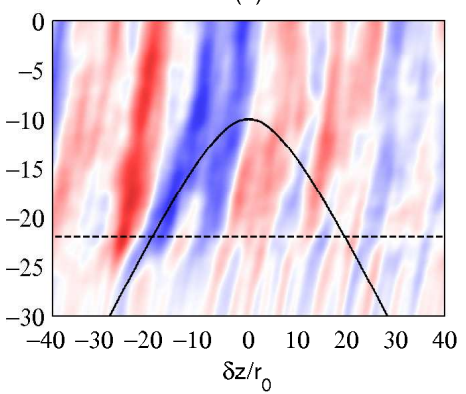

(c)

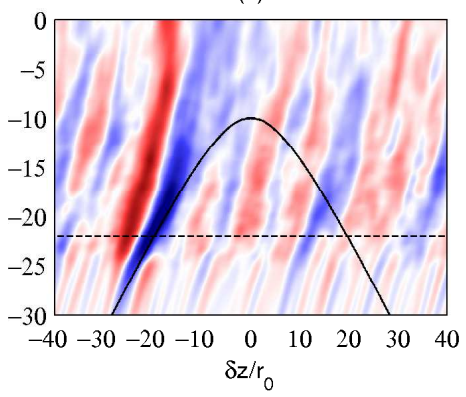

(e)
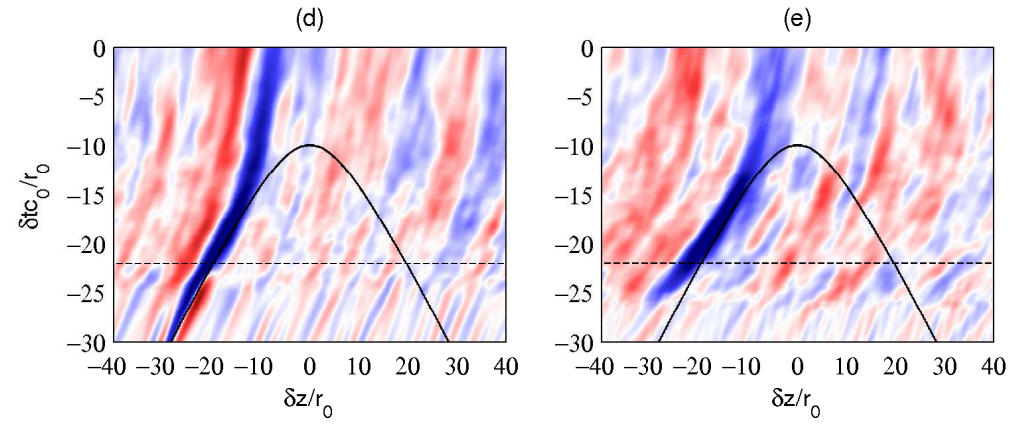

Figure 10. Space-time cross-correlations obtained between pressure fluctuations at $r=10 r_{0}$ and $t=t_{c}+$ $22 r_{0} / c_{0}$ and axial velocity fluctuations $u_{z}^{\prime}$ at $r=0$ and time $t-\delta t$ for (a) $\mathrm{M}=0.3$, (b) $\mathrm{M}=0.6,(\mathrm{c}) \mathrm{M}=0.9$, (d) $M=1.3$ and (e) $M=2$. The color scale ranges from -0.20 to 0.20 , from blue to red; - propagation at the ambient speed of sound; $--t=t_{c}$.

(a)

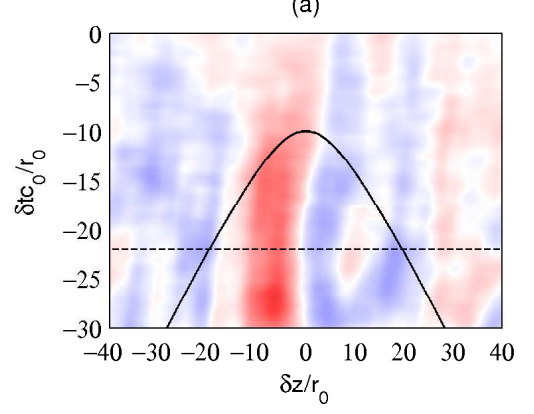

(b)

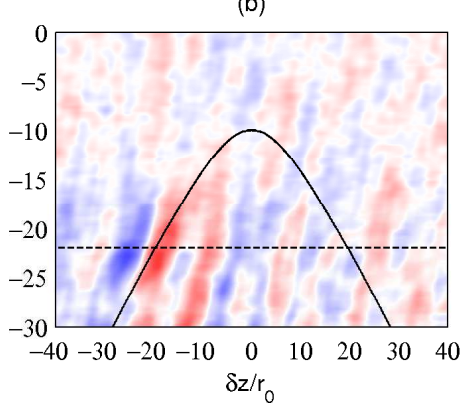

(c)

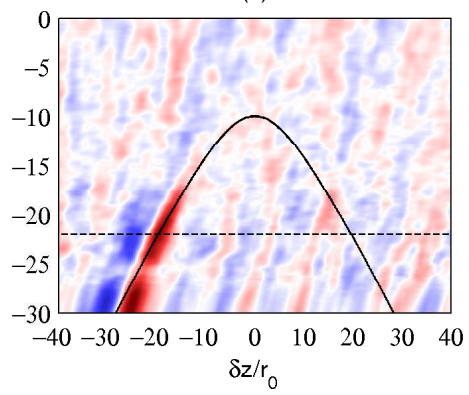

(e)
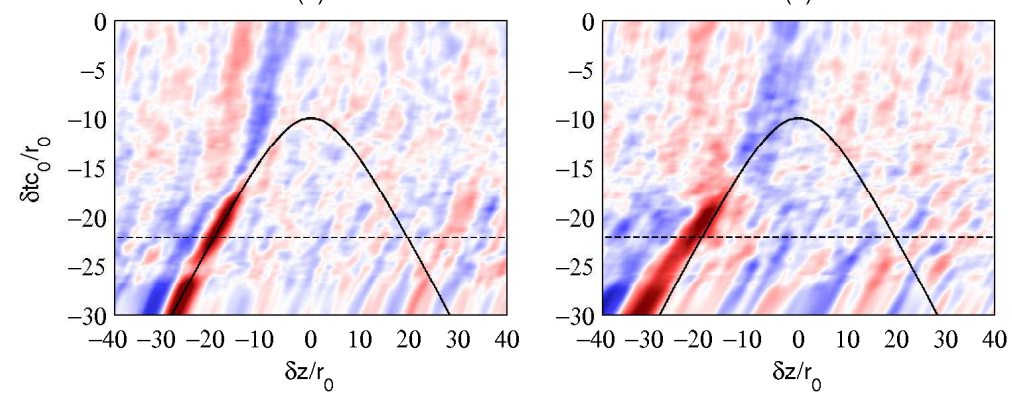

Figure 11. Space-time cross-correlations obtained between pressure fluctuations at $r=10 r_{0}$ and $t=t_{c}+$ $22 r_{0} / c_{0}$ and vorticity fluctuations $|\omega|^{\prime}$ at $r=0$ and time $t-\delta t$ for $(\mathrm{a}) \mathrm{M}=0.3,(\mathrm{~b}) \mathrm{M}=0.6,(\mathrm{c}) \mathrm{M}=0.9$, (d) $M=1.3$ and (e) $M=2$. The color scale ranges from -0.20 to 0.20 , from blue to red; at the ambient speed of sound; $--t=t_{c}$. 
(a)

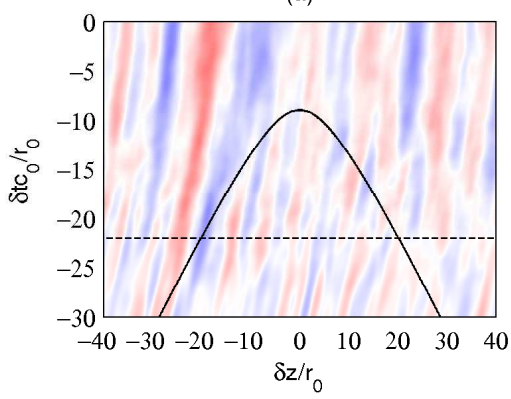

(b)

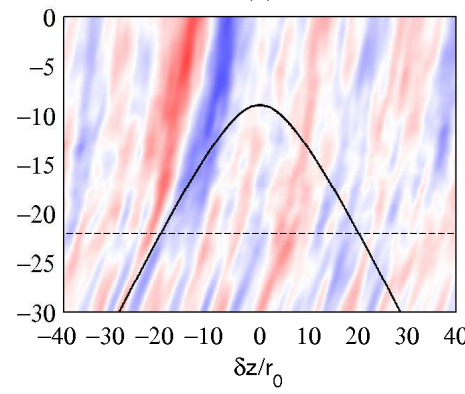

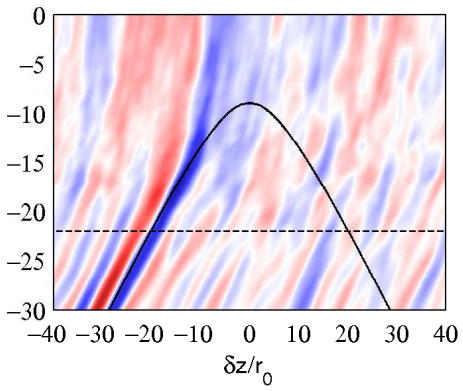

Figure 12. Space-time cross-correlations obtained between pressure fluctuations at $r=10 r_{0}$ and $t=t_{c}+$ $22 r_{0} / c_{0}$ and axial velocity fluctuations $u_{z}^{\prime}$ at $r=r_{0}$ and time $t-\delta t$ for (a) $\mathrm{M}=0.9$, (b) $\mathrm{M}=1.3$ and (c) $\mathrm{M}=2$. The color scale ranges from -0.20 to 0.20 , from blue to red; - propagation at the ambient speed of sound; $--t=t_{c}$.

(a)

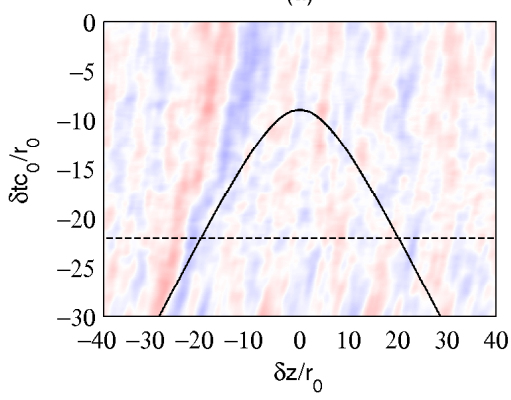

(b)

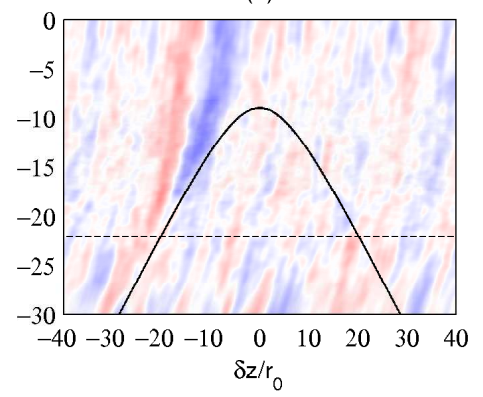

(c)

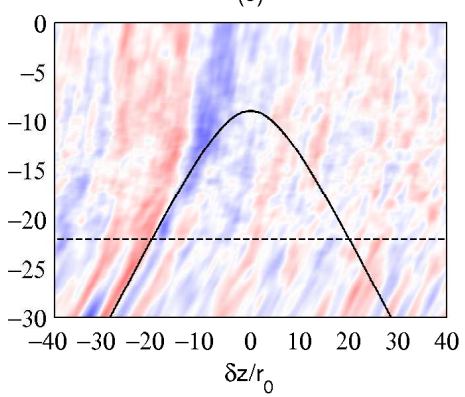

Figure 13. Space-time cross-correlations obtained between pressure fluctuations at $r=10 r_{0}$ and $t=t_{c}+$ $22 r_{0} / c_{0}$ and vorticity fluctuations $|\omega|^{\prime}$ at $r=r_{0}$ and time $t-\delta t$ for (a) $M=0.9,(b) M=1.3$ and $(\mathrm{c}) \mathrm{M}=2$. The color scale ranges from -0.20 to 0.20 , from blue to red; — propagation at the ambient speed of sound; $--t=t_{c}$.

flow fluctuations, whose values are significant and increase with the jet Mach number as in experiments for spatial jets. However, there is no clear evidence of such a radiation for the jet at $\mathrm{M}=0.3$. Moreover, Mach waves also appear to be generated in the mixing layers of the jet at $\mathrm{M}=2$.

The results of the present work suggest the existence, in temporal jets for Mach numbers ranging from 0.6 to 2 , of a common mechanism emitting noise in the downstream direction mainly when the potential core closes. This mechanism seems to be efficient in high-subsonic and supersonic jets, as is the case for the source assumed to responsible for the low-frequency downstream noise component of spatially-developing jets. $^{3}$ Further studies are required to identify the nature of this mechanism unambiguously. For the present temporal jet at a Mach number of 0.9 , the use of conditional averaging allowed us to extract some of its features. ${ }^{12}$ The generation of noise around the time of potential-core closing was found to be related to the process of growth and decay of instability waves in the jet flow. The presence of such a process, likely to radiate noise even in subsonic jets, will be examined in the other temporal jets.

\section{Acknowledgments}

This work was granted access to the HPC resources of FLMSN (Fédération Lyonnaise de Modélisation et Sciences Numériques), partner of EQUIPEX EQUIP@MESO, and of the resources of IDRIS (Institut du Développement et des Ressources en Informatique Scientifique) under the allocation 2017-2a0204 made by GENCI (Grand Equipement National de Calcul Intensif). It was performed within the framework of the Labex CeLyA of Université de Lyon, operated by the French National Research Agency (Grant No. ANR-10-LABX-0060/ANR-11-IDEX-0007). 


\section{References}

${ }^{1}$ Tam, C. K. W., "Supersonic jet noise," Annu. Rev. Fluid Mech., Vol. 27, 1995, pp. 17-43.

${ }^{2}$ Mollo-Christensen, E., Kolpin, M. A., and Martucelli, J. R., "Experiments on jet flows and jet noise far-field spectra and directivity patterns," J. Fluid Mech., Vol. 18, 1964, pp. 285-301.

${ }^{3}$ Tam, C. K. W., Viswanathan, K., Ahuja, K. K., and Panda, J., "The sources of jet noise: experimental evidence," J. Fluid Mech., Vol. 615, 2008, pp. 253-292.

${ }^{4}$ Tam, C. K. W., "Jet noise: Since 1952," Theor. Comput. Fluid Dyn., Vol. 10, No. 1-4, 1998, pp. 393-405.

${ }^{5} \mathrm{Chu}$, W. T. and Kaplan, R. E., "Use of a spherical concave reflector for jet-noise-source distribution diagnosis," J. Acoust. Soc. Am., Vol. 59, No. 6, 1976, pp. 1268-1277.

${ }^{6}$ Fisher, M. J., Harper-Bourne, M., and Glegg, S. A. L., "Jet engine noise source location: The polar correlation technique," J. Sound Vib., Vol. 51, No. 1, 1977, pp. 23-54.

${ }^{7}$ Lee, S. S. and Bridges, J., "Phased-array measurements of single flow hot jets," NASA/TM 2005-213826, 2005.

${ }^{8}$ Lee, H. K. and Ribner, H. S., "Direct correlation of noise and flow of a jet," J. Acoust. Soc. Am., Vol. 52, No. 5, 1972, pp. $1280-1290$.

${ }^{9}$ Schaffar, M., "Direct measurements of the correlation between axial in-jet velocity fluctuations and far field noise near the axis of a cold jet," J. Sound Vib., Vol. 64, No. 1, 1979, pp. 73-83.

${ }^{10}$ Panda, J., Seasholtz, R. G., and Elam, K. A., "Investigation of noise sources in high-speed jets via correlation measurements," J. Fluid Mech., Vol. 537, 2005, pp. 349-385.

${ }^{11}$ Bogey, C. and Bailly, C., "An analysis of the correlations between the turbulent flow and the sound pressure field of subsonic jets," J. Fluid Mech., Vol. 583, 2007, pp. 71-97.

${ }^{12}$ Bogey, C., "On the noise generated by the potential-core closing of temporally-developing subsonic jets," AIAA Paper 2017-3851, 2017.

${ }^{13}$ Fortuné, V., Lamballais, E., and Gervais, Y., "Noise radiated by a non-isothermal, temporal mixing layer. Part I: Direct computation and prediction using compressible DNS," Theor. Comput. Fluid Dyn., Vol. 18, No. 1, 2004, pp. 61-81.

${ }^{14}$ Kleimann, R. R. and Freund, J. B., "The sound from mixing layers simulated with different ranges of turbulence scales," Phys. Fluids, Vol. 20, No. 10, 2008, pp. 101503.

${ }^{15}$ Buchta, D. A. and Freund, J. B., "The role of large-scale structures on crackle noise," AIAA Paper 2016-3027, 2016.

${ }^{16}$ Terakado, D., Nonomura, T., Oyama, A., and Fujii, K., "Mach number dependence on sound sources in high Mach number turbulent mixing layer," AIAA Paper 2016-3015, 2016.

${ }^{17}$ Lighthill, M. J., "On sound generated aerodynamically I. General theory," Proc. R. Soc. Lond. A, Vol. 211, No. 1107, 1952, pp. 564-587.

${ }^{18}$ Ffowcs Williams, J. E., "The noise from turbulence convected at high speed," Phil. Trans. R. Soc. Lond. A, Vol. 255, No. 1061, 1963, pp. 469-503.

${ }^{19}$ Bogey, C., Barré, S., Fleury, V., Bailly, C., and Juvé, D., "Experimental study of the spectral properties of near-field and far-field jet noise," Int. J. Aeroacoust., Vol. 6, No. 2, 2007, pp. 73-92.

${ }^{20}$ Zaman, K. B. M. Q. and Yu, J. C., "Power spectral density of subsonic jet noise," J. Sound Vib., Vol. 98, No. 4, 1985, pp. 519-537.

${ }^{21}$ Ahuja, K. and Bushell, K., "An experimental study of subsonic jet noise and comparison with theory," J. Sound Vib., Vol. 30, No. 3, 1973, pp. 317-341.

${ }^{22}$ Bogey, C., "Direct numerical simulation of a temporally-developing subsonic round jet and its sound field," AIAA Paper 2017-0925, 2017.

${ }^{23}$ Pineau, P. and Bogey, C., "Numerical study of temporally-developing supersonic round jets and their sound fields," AIAA Paper 2017-3209, 2017.

${ }^{24}$ Zaman, K. B. M. Q., "Effect of initial condition on subsonic jet noise," AIAA J., Vol. 23, No. 9, 1985, pp. $1370-1373$.

${ }^{25}$ Bogey, C., Bailly, C., and Juvé, D., "Noise investigation of a high subsonic, moderate Reynolds number jet using a compressible LES," Theor. Comput. Fluid Dyn., Vol. 16, No. 4, 2003, pp. 273-297.

${ }^{26}$ Bogey, C., Marsden, O., and Bailly, C., "Influence of initial turbulence level on the flow and sound fields of a subsonic jet at a diameter-based Reynolds number of $10^{5}$," J. Fluid Mech., Vol. 701, 2012, pp. 352-385.

${ }^{27}$ Bogey, C., Marsden, O., and Bailly, C., "Large-Eddy Simulation of the flow and acoustic fields of a Reynolds number $10^{5}$ subsonic jet with tripped exit boundary layers," Phys. Fluids, Vol. 23, No. 3, 2011, pp. 035104.

${ }^{28}$ Bogey, C. and Marsden, O., "Simulations of initially highly disturbed jets with experiment-like exit boundary layers," AIAA J., Vol. 54, No. 4, 2016, pp. 1299-1312.

${ }^{29}$ Bogey, C., "Grid sensitivity of flow field and noise of high-Reynolds-number jets computed by large-eddy simulation," Int. J. Aeroacoust., Vol. 17, No. 4-5, 2018.

${ }^{30}$ Mohseni, K. and Colonius, T., "Numerical treatment of polar coordinate singularities," J. Comput. Phys., Vol. 157, No. 2, 2000, pp. 787-795.

${ }^{31}$ Bogey, C., de Cacqueray, N., and Bailly, C., "Finite differences for coarse azimuthal discretization and for reduction of effective resolution near origin of cylindrical flow equations," J. Comput. Phys., Vol. 230, No. 4, 2011, pp. 1134-1146.

${ }^{32}$ Bogey, C. and Bailly, C., "A family of low dispersive and low dissipative explicit schemes for flow and noise computations," J. Comput. Phys., Vol. 194, No. 1, 2004, pp. 194-214.

${ }^{33}$ Berland, J., Bogey, C., Marsden, O., and Bailly, C., "High-order, low dispersive and low dissipative explicit schemes for multi-scale and boundary problems," J. Comput. Phys., Vol. 224, No. 2, 2007, pp. 637-662.

${ }^{34}$ Bogey, C. and Bailly, C., "Influence of nozzle-exit boundary-layer conditions on the flow and acoustic fields of initially laminar jets," J. Fluid Mech., Vol. 663, 2010, pp. 507-539. 
${ }^{35}$ Tam, C. K. W. and Dong, Z., "Radiation and outflow boundary conditions for direct computation of acoustic and flow disturbances in a nonuniform mean flow," J. Comput. Acoust., Vol. 4, No. 2, 1996, pp. $175-201$.

${ }^{36}$ Bogey, C. and Bailly, C., "Three-dimensional non reflective boundary conditions for acoustic simulations: far-field formulation and validation test cases," Acta Acustica, Vol. 88, No. 4, 2002, pp. 463-471.

${ }^{37}$ Michalke, A., "On the inviscid instability of the hyperbolic-tangent velocity profile," J. Fluid Mech., Vol. 19, No. 4, 1964, pp. 543-556.

${ }^{38}$ Arndt, R. E. A., Long, D. F., and Glauser, M. N., "The proper orthogonal decomposition of pressure fluctuations surrounding a turbulent jet," J. Fluid Mech., Vol. 340, 1997, pp. 1-33.

${ }^{39}$ Coiffet, F., Jordan, P., Delville, J., Gervais, Y., and Ricaud, F., "Coherent structures in subsonic jets: a quasi-irrotational source mechanism?" Int. J. Aeroacoust., Vol. 5, No. 1, 2005, pp. 67-89.

${ }^{40}$ Bogey, C. and Bailly, C., "Investigation of downstream and sideline subsonic jet noise using Large Eddy Simulations," Theor. Comput. Fluid Dyn., Vol. 20, No. 1, 2006, pp. 23-40.

${ }^{41}$ Michalke, A., "Survey on jet instability theory," Prog. Aerospace Sci., Vol. 21, 1984, pp. $159-199$.

${ }^{42}$ Brown, G. L. and Roshko, A., "On density effects and large structure in turbulent mixing layers," J. Fluid Mech., Vol. 64, No. 4, 1974, pp. 775-816.

${ }^{43}$ Seiner, J. M., The distribution of jet source strength intensity by means of direct correlation technique, Ph.D. thesis, Pennsylvania State University, 81974.

${ }^{44}$ Panda, J., "Experimental investigation of turbulent density fluctuations and noise generation from heated jets," J. Fluid Mech., Vol. 591, 2007, pp. 73-96.

${ }^{45}$ Bogey, C., Barré, S., Juvé, D., and Bailly, C., "Simulation of a hot coaxial jet : direct noise prediction and flow-acoustics correlations," Phys. Fluids, Vol. 21, No. 3, 2009, pp. 035105.

${ }^{46}$ Grizzi, S. and Camussi, R., "Wavelet analysis of near-field pressure fluctuations generated by a subsonic jet," J. Fluid Mech., Vol. 698, 2012, pp. 93-124.

${ }^{47}$ Henning, A., Koop, L., and Schröder, A., "Causality correlation analysis on a cold jet by means of simultaneous Particle Image Velocimetry and microphone measurements," J. Sound Vib., Vol. 332, 2013, pp. 3148-3162. 